\title{
INFLUENCIA DEL GÉNERO SOBRE EL PROCESO DE DESARROLLO DEL SÍNDROME DE QUEMARSE POR EL TRABAJO (BURNOUT) EN PROFESIONALES DE ENFERMERÍA
}

\begin{abstract}
RESUMEN. El objetivo de este estudio es analizar si el género establece diferencias significativas en los niveles y en el proceso del síndrome de quemarse por el trabajo. Los resultados obtenidos indican que los hombres $(\mathrm{N}=72)$ puntúan significativamente más alto que las mujeres $(\mathrm{N}=258)$ en despersonalización. En la muestra de hombres la realización personal en el trabajo no resultó un predictor significativo de despersonalización. En la muestra de mujeres el agotamiento emocional no predijo significativamente la inclinación al absentismo. Se concluye sobre la importancia de que los gestores de personal en sanidad tengan presente la diferencia de género.
\end{abstract}

Palabras-clave:Quemarse por el trabajo, estrés laboral, enfermería.

\section{THE INFLUENCE OF GENDER ON THE DEVELOPMENT OF BURNOUT SYNDROME PROCESS IN NURSING PROFESSIONALS}

\begin{abstract}
The purpose of this study was to analyze if gender differences establish significant differences in the levels and process of the burnout syndrome. Results indicate that the men $(\mathrm{N}=72)$ perceive significantly more despersonalization that the women $(\mathrm{N}=258)$. In the sample of men personal accomplishment was not a significant antecedent of despersonalization, while in the sample of women emotional exhaustion was not a significant antecedent of inclination to be absent. As a conclusion it is recommended that managers of personnel in health sector should take into consideration the gender differences in the intervention on the burnout syndrome.
\end{abstract}

Key words: Burnout syndrome, job stress, nurses.

El concepto de "quemarse por el trabajo" (burnout) surgió en Estados Unidos a mediados de los años 70 (Freudenberguer, 1974) para explicar el proceso de deterioro en los cuidados y atención profesional a los usuarios de las organizaciones de servicios (organizaciones de voluntariado, sanitarias, de servicios sociales, educativas, etc.). A lo largo de estos años se ha establecido que el síndrome de quemarse por el trabajo es una respuesta al estrés laboral crónico que, aunque se puede desarrollar en todo tipo de profesionales, ocurre con relativa frecuencia en los profesionales de las organizaciones de servicios que trabajan en contacto directo con los usuarios de esas organizaciones, y deteriora significativamente su calidad de vida laboral y consecuentemente la calidad del servicio que ofrece la organización.

En España, el estrés laboral y el síndrome de quemarse por el trabajo ha adquirido una relevancia especial en la atención que dedican académicos (v.g., Moreno, 2000), profesionales y sindicatos a la prevención de los riesgos laborales (v.g., FOREMCCOO; 2000; SATSE, 1999), y es noticia y objeto de continuo debate en medios de comunicación, donde se insiste sobre la necesidad de reconocerlo como enfermedad profesional, y sobre la importancia que tiene abordar su prevención y tratamiento. Un paso

\footnotetext{
Associate Professor of Work and Organizational Psychology. Departamento de Psicobiología y Psicología Social, Facultad de Psicología, Universidad de Valencia.

Endereço para correspondência: Avda. Blasco Ibáñez, 21, 46010, Valencia, España. E-mail: Pedro.Gil-Monte@uv.es
} 
relevante en este sentido ha sido la sentencia dictada el 26 de octubre del 2000 por el Tribunal Supremo, Sala de lo Social, que lo reconoce como accidente laboral.

Gil-Monte, Peiró y Valcárcel (1998) han concluido que esta respuesta al estrés laboral crónico es un proceso que se inicia con el desarrollo de ideas sobre fallo profesional y con el desarrollo de actitudes negativas hacia el rol profesional (falta de realización personal en el trabajo) junto a sentimientos de encontrarse emocionalmente agotado (agotamiento emocional), y posteriormente los individuos generan actitudes y sentimientos negativos hacia las personas con las que se trabaja (actitudes de despersonalización). Este modelo supone una alternativa teórica y empírica a otros modelos elaborados para explicar el desarrollo del proceso de quemarse por el trabajo (Golembiewski, Munzenrider y Carter, 1983; Lee y Ashforth, 1993a, 1993b; Leiter y Maslach, 1988), y ha obtenido confirmación empírica en diferentes estudios (Manassero, García, Vázquez, Ferrer, Ramis y Gili, 2000; Manzano y Ramos, 2000).

La profesión de enfermería es una ocupación que por sus singulares características (escasez de personal que supone sobrecarga laboral, trabajo en turnos, relación con pacientes y familiares problemáticos, contacto directo con la enfermedad, el dolor y la muerte, falta de reconocimiento profesional, falta de autonomía y autoridad en el trabajo para poder tomar decisiones, etc.) genera estrés laboral crónico, y es una de las ocupaciones donde mayor incidencia tiene el síndrome de quemarse por el trabajo. Sus consecuencias más relevantes son el deterioro de la calidad de los servicios que ofrecen las instituciones sanitarias y el alto índice de absentismo que existe entre estos profesionales.

La sobrecarga laboral tiene una incidencia especial como fuente de estrés crónico en enfermería. Estudios empíricos cuantitativos han obtenido relaciones significativas entre sobrecarga de trabajo y los síntomas del síndrome de quemarse por el trabajo estimado con el Maslach Burnout Inventory (MBI) (Maslach y Jackson, 1986) en profesionales de enfermería (Bacharach, Bamberger y Conley, 1991; Janssen, Schaufeli, y Houkes, 1999; Leiter, 1991; Novak y Chappell, 1996; Richardsen, Burke y Leiter, 1992; Zellars, Perrewé y Hochwarter, 2000; Zohar, 1997). Richardsen et al. (1992) obtuvieron que la carga de trabajo era uno de los principales predictores de los sentimientos de agotamiento emocional. Pero además, si los profesionales perciben que carecen de las destrezas y habilidades necesarias para realizar las tareas (sobrecarga de trabajo cualitativa) probablemente desarrollarán baja realización personal en el trabajo. En general, los estudios han obtenido que existe una tendencia según la cual la relación entre sobrecarga de trabajo y agotamiento emocional resulta más intensa que la relación entre sobrecarga y las otras dimensiones del MBI. En un estudio metaanalítico Lee y Ashforth (1996) obtuvieron una correlación media de .65 para la relación entre sobrecarga y agotamiento emocional, la correlación resultó de -.09 (n.s.) con realización personal en el trabajo, y .34 con despersonalización. Janssen et al. (2000) obtuvieron con un modelo causal que la sobrecarga es un predictor significativo de agotamiento emocional, pero no de realización personal en el trabajo, ni de despersonalización.

Otra de las fuentes de estrés crónico en profesionales de enfermería es la percepción de falta de reciprocidad en los intercambios. En las organizaciones de servicios, el síndrome de quemarse por el trabajo surge primordialmente en un contexto social, por ello las teorías del intercambio social (ver, Donnerstein y Hatfield, 1982; Kelley, 1979; McClintock y Keil, 1982) pueden ser de fundamental importancia para entender la aparición del síndrome y su relación con la interacción social en las organizaciones. Los profesionales de enfermería están implicados en relaciones con los pacientes, y en esas relaciones los procesos de intercambio social y las expectativas de reciprocidad juegan un papel muy importante. Varios estudios han obtenido que existe una relación positiva y significativa entre la percepción de falta de reciprocidad en los intercambios sociales y las dimensiones del MBI. Esta relación resulta positiva con agotamiento emocional y con despersonalización, y negativa con realización personal en el trabajo (Bakker, Schaufeli, Sixma, Bosveld y Van Dierendonck, 2000; Buunk y Schaufeli, 1993; Schaufeli, y Janczur, 1994). En relación a programas de intervención, tras desarrollar un programa para prevenir el absentismo, y a la luz de los resultados obtenidos en profesionales de la salud, Van Dierendonck, Schaufeli y Buunk (1998) concluyeron que los sentimientos de inequidad en la relación con la organización es una variable especialmente importante a considerar en el diseño de programas de intervención sobre el síndrome de quemarse por el trabajo y al absentismo laboral.

El absentismo es un problema serio en la ocupación de enfermería por la tasa relativamente alta que presenta, y aparece como una de las principales consecuencias del síndrome de quemarse por el trabajo, pues las dimensiones del síndrome aparecen asociadas significativamente con actitudes y conductas de absentismo. Desde una perspectiva correlacional, 
algunos estudios han obtenido que los sentimientos de agotamiento emocional se asocian significativamente de manera positiva con el número de faltas en el trabajo en profesionales de enfermería (Firth y Britton, 1989) y, en diseño longitudinal, Iverson, Olekalns y Erwin (1998) obtuvieron mediante un modelo causal que la realización personal en el trabajo era un predictor significativo de la tasa de absentismo evaluada 6 meses después (beta $=-.18$ ).

Por otra parte, algunos estudios y organizaciones del mundo del trabajo subrayan la necesidad de considerar las diferencias de género a la hora de realizar diagnóstico e intervención en las organizaciones, pues esta variable puede establecer diferencias significativas en los procesos que se desarrollan en los contextos laborales, especialmente en aquellos vinculados a la salud y calidad de vida de los trabajadores (v.g., Bureau Technique Syndical Européen pour la Santé et la Sécurité, BTS; Sociedad Española de Medicina y Seguridad en el Trabajo).

Diferentes estudios han obtenido que existen diferencias significativas en los niveles de algunas de las dimensiones del síndrome de quemarse por el trabajo en función del género. La tendencia general de los resultados es obtener diferencias significativas en función del sexo en los niveles de despersonalización en el sentido de que los varones puntúan significativamente más alto que las mujeres en esta dimensión (para una revisión ver Gil-Monte, Peiró y Valcárcel, 1996). La explicación a estas diferencias puede residir en la consideración tradicional de la profesión de enfermería como una profesión femenina, estrechamente relacionada con la feminización del cuidado. Sin embargo, en las últimas décadas hemos asistido a un crecimiento del número de varones que optan por esta profesión. No obstante, su elección por parte de los varones no se corresponde con cambios en los modelos de socialización ni con el cambio de la imagen tradicional de la profesión, sino más bien a otro tipo de cuestiones tales como la accesibilidad al empleo, la corta duración en los estudios y la inclusión de los mismos en los catálogos de titulaciones universitarias. Esta situación también puede dar lugar a diferentes modelos en el desarrollo del síndrome de quemarse por el trabajo (relación antecedentes y consecuentes del síndrome con sus dimensiones) en los profesionales de enfermería, pero como las muestras que componen los estudios con estos profesionales están integradas en su mayor parte por mujeres la influencia de la variable género en el proceso de quemarse por el trabajo puede estar enmascarada.

Es por ello que el primer objetivo de este estudio es identificar si en función del género existen diferencias significativas en las dimensiones del MBI, $\mathrm{y}$ en sus antecedentes y consecuencias consideradas en este estudio. El segundo objetivo consiste en explorar si en base al género se establecen diferencias significativas en el padrón de desarrollo del proceso de quemarse por el trabajo. Con base en los argumentos expresados arriba, se hipotetiza que los hombres de la muestra percibirán mayores niveles significativos de sobrecarga laboral y de falta de reciprocidad en los intercambios, sus puntuaciones serán significativamente mayores en los niveles de despersonalización y en la inclinación al absentismo. Por otra parte, y en relación al desarrollo del proceso de quemarse por el trabajo, se asume a priori que las relaciones hipotetizadas entre las variables que intervienen en el estudio son idénticas para ambos géneros. Estas relaciones aparecen hipotetizadas en el modelo que se presenta en el Figura 1.

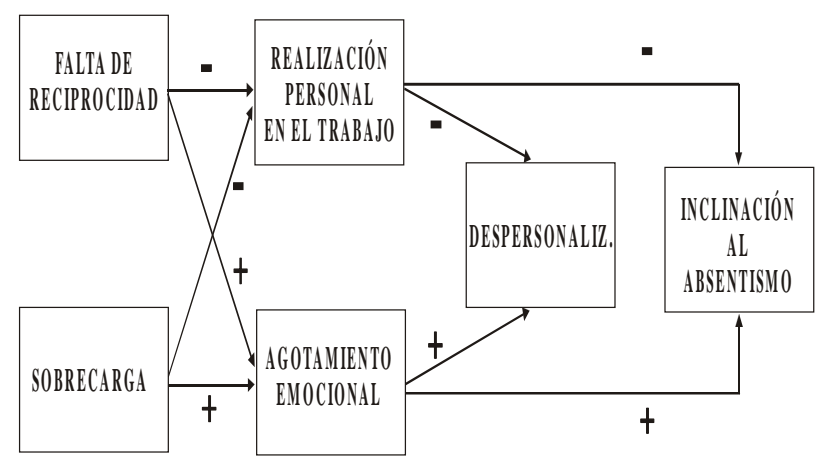

Figura 1. Modelo hipotetizado.

\section{MÉTODO}

\section{Muestra}

La muestra del estudio está compuesta por 330 sujetos. 72 sujetos se identificaron como hombres $(21.8 \%)$ y 258 como mujeres $(78.2 \%)$. La media de edad de la muestra fue $\mathrm{M}=31.8(\mathrm{dt}=7.27$, $\max .=58$, min. $=20$, Mo $=25$ ). Según el tipo de contrato, 235 sujetos eran fijos (71.2\%) y 94 eventuales $(28.5 \%)$.

\section{Instrumentos}

El sexo fue evaluado mediante un ítem de dos alternativas en el que el sujeto debía redondear la opción del sexo que le incluía (hombre vs. mujer). La Sobrecarga laboral percibida fue medida con siete ítems del "Organizational Stress Questionnaire" (OSQ) (Caplan, Cobb, French, Van Harrison y Pinneau, 1975) ( $\alpha=.79)$. La Falta de Reciprocidad fue evaluada mediante cinco ítems (v.g.: "Pongo más en mi trabajo de lo que obtengo a cambio de él"). Los 
sujetos respondieron a esos ítems con una escala de cinco grados que va de "Totalmente de acuerdo" (1) a "Totalmente en desacuerdo" (5) ( $\alpha=$.79). El síndrome de quemarse por el trabajo se estimó mediante el MBI (Maslach y Jackson, 1986). Este instrumento está compuesto por veintidos ítems que miden las tres dimensiones del síndrome: Realización Personal en el Trabajo (PA) (8 ítems) $(\alpha=.69)$, Agotamiento Emocional (EE) $(9$ ítems $) \quad(\alpha=.87), \quad \mathrm{y}$ Despersonalización (D) (5 ítems) $(\alpha=.59)$. La Inclinación al Absentismo se evaluó mediante 4 ítems (v.g., "Comunicaría que se encuentra enfermo para no ir a trabajar por importantes razones privadas"). Estos items se evalúan con una escala Likert de cinco grados que va "Siempre" (1) a "Nunca"(5) ( $\alpha=.71)$.

\section{Procedimiento}

Los datos fueron recogidos por el autor seleccionando a los sujetos de manera no aleatoria en dos hospitales integrados en el Servicio Canario de Salud, y ubicados en la ciudad de Santa Cruz de Tenerife (España). En relación al número de camas, los recursos de infraestructura son superiores a las 650 unidades en cada uno de los dos hospitales. Aunque la ciudad en la que se encuentran los centros cuenta con una población superior a los 214.000 habitantes, la población del área de referencia es aproximadamente de 470.000 habitantes. Dado que la muestra del presente estudio está integrada por la unión de las respuestas emitidas por los sujetos de diferentes estudios, los porcentajes de respuesta son diferentes, situándose en torno al $30 \%$.

\section{RESULTADOS}

Como se aprecia en la diagonal de la Tabla 2, los valores de consistencia interna alcanzados fueron aceptables para todas las escalas, excepto para la escala de despersonalización que presentó un valor alfa de Cronbach relativamente bajo $(\alpha=.59)$.

En relación a las medias obtenidas en los antecedentes y consecuentes del síndrome por los subgrupos de las muestra (ver Tabla 1) cabe señalar que los hombres puntúan más alto significativamente que las mujeres en Falta de reciprocidad (3.52 vs. 3.28 , t-test $=2.21, \mathrm{p}<.05)$, e Inclinación al absentismo $(1.88$ vs. $1.68, \mathrm{t}$-test $=2.25, \mathrm{p}<.05)$. El valor $\mathrm{t}$-test para la diferencia de medias en las dimensiones del MBI, sólo resultó significativo para la dimensión de despersonalización, en el sentido de que la media de los hombres resultó significativamente superior a la de las mujeres $(8.05$ vs. 5.91 , t-test $=2.93, \mathrm{p}<.01)$. En relación al primer objetivo, estos resultados confirman la hipótesis formulada para todas las variables con excepción de la sobrecarga laboral.

Tabla 1. Estadísticos descriptivos para las variables del estudio, para el total de la muestra y por subgrupos según género.

\begin{tabular}{|c|c|c|c|c|c|c|}
\hline & \multicolumn{3}{|c|}{ M } & \multicolumn{3}{|c|}{ dt } \\
\hline & $\begin{array}{c}\text { Total } \\
(\mathrm{N}=\mathbf{3 3 0})\end{array}$ & $\begin{array}{c}\text { Hombre } \\
(\mathrm{N}=72)\end{array}$ & $\begin{array}{c}\text { Mujer } \\
\text { (N=258) }\end{array}$ & $\begin{array}{c}\text { Total } \\
(\mathbf{N}=\mathbf{3 3 0})\end{array}$ & $\begin{array}{c}\text { Hombre } \\
(\mathbf{N}=72)\end{array}$ & $\begin{array}{c}\text { Mujer } \\
(\mathbf{N}=\mathbf{2 5 8})\end{array}$ \\
\hline 1. Sobrecarga & 3.38 & 3.29 & 3.40 & .64 & .63 & .64 \\
\hline 2. Falta de reciprocidad & 3.33 & 3.52 & 3.28 & .75 & .68 & .77 \\
\hline 3. Realización personal & 36.81 & 35.82 & 37.09 & 6.15 & 6.86 & 5.92 \\
\hline $\begin{array}{l}\text { 4. Agotamiento } \\
\text { emocional }\end{array}$ & 23.91 & 24.97 & 23.62 & 11.26 & 11.50 & 11.20 \\
\hline 5. Despersonalización & 6.38 & 8.06 & 5.91 & 5.05 & 5.68 & 4.77 \\
\hline $\begin{array}{l}\text { 6. Inclinación } \\
\text { absentismo }\end{array}$ & 1.73 & 1.88 & 1.68 & .67 & .68 & .66 \\
\hline
\end{tabular}

Para explorar la segunda parte del objetivo planteado, esto es, si se establecen padrones diferentes en el proceso de desarrollo del síndrome de quemarse por el trabajo en función del género, se elaboró un modelo causal que se probó alternativamente en las dos muestras del estudio: hombres vs. mujeres. La matriz de correlaciones $r$ de Pearson entre las variables que intervienen en el modelo se encuentra en la Tabla 2 .

Tabla 2. Matriz de correlaciones $\mathrm{r}$ de Pearson entre las variables del estudio.

\begin{tabular}{|c|c|c|c|c|c|c|}
\hline & 1 & 2 & 3 & 4 & 5 & 6 \\
\hline 1. Sobrecarga & $(.79)$ & $.37 * *$ & .08 & $.32 * *$ & .20 & .13 \\
\hline 2. Falta de reciprocidad & $.24 * * *$ & $(.79)$ & -.21 & $.48^{* * *}$ & $.27 *$ & $.35^{* *}$ \\
\hline 3. PA & -.06 & $-.31 * * *$ & $(.69)$ & $-.33 * *$ & $-.32 * *$ & $-.42 * * *$ \\
\hline 4. EE & $.32 * * *$ & $.45 * * *$ & $-.34 * * *$ & $(.87)$ & $.57 * * *$ & $.47 * * *$ \\
\hline 5. D & $.22 * * *$ & $.21^{* * *}$ & $-.36 * * *$ & $.42 * * *$ & $(.59)$ & $.24 *$ \\
\hline 6. Inclinación al absentismo & -.05 & .10 & $-.20 * * *$ & $.13^{*}$ & $.14^{*}$ & $(.71)$ \\
\hline
\end{tabular}

El modelo causal fue analizado con el programa LISREL 8 (Jöreskog y Sörbom, 1996) y el método de estimación utilizado fue Maximum Likelihood. En ambas muestras el modelo presentó un ajuste adecuado a los datos a través de diferentes índices (ver Tabla 3). Para la muestra de hombres, el ajuste del modelo resultó adecuado según todos los índices de ajuste considerados (valores inferiores a 2 en $\chi^{2} / \mathrm{gl}$ indican que el modelo es plausible, Byrne,1992). Para la muestra de mujeres, el modelo también alcanzó un ajuste adecuado a través del índice GFI (Bentler, 1992) y CFI (Hoyle, 1995), aunque el ajuste no fue adecuado según el resto de índices (Browne y Cudeck, 1993; Hair, Anderson, Tatham y Black, 1995; Marsh, Balla y Hau, 1996). 
Tabla 3. Índices de ajuste para todos los modelos del estudio.

\begin{tabular}{lcccccccc}
\hline & $\chi^{\mathbf{2}}$ & gl & $\chi^{\mathbf{2}} /$ gl & GFI & RMSEA & CFI & \multicolumn{2}{c}{ NNFI PNFI } \\
\cline { 2 - 9 } Hombres inicial & 8.13 & 6 & 1.35 & .96 & .072 & .98 & .94 & .37 \\
Mujeres inicial & $25.24^{*}$ & 6 & 4.21 & .97 & .110 & .91 & .76 & .35 \\
\hline Hombres revisado & 10.66 & 8 & 1.33 & .96 & .069 & .97 & .94 & .48 \\
Mujeres revisado $28.07^{*}$ & 8 & 3.51 & .97 & .099 & .90 & .82 & .47 \\
\hline
\end{tabular}

Nota 1. GFI = Goodness Fit Index, RMSEA $=$ Root Mean Square Error of Approximation, $\mathrm{CFI}=$ Comparative Fit Index, NNFI $=$ Non-Normed Fit Index, PNFI $=$ Parsimony Normed Fit Index. $* \mathrm{p}<.001$

Al analizar las relaciones que se establecen entre las variable del modelo se observó que para la muestra de hombres la relación entre Sobrecarga y Realización Personal en el Trabajo $(\gamma=.14, \quad \mathrm{t}=1.10), \mathrm{y}$ entre ésta $\mathrm{y}$ Despersonalización $(\beta=-.12, \mathrm{t}=-1.18)$ no resultaron significativas. Para la muestra de mujeres tampoco resultó significativa la relación entre Sobrecarga y Realización Personal en el Trabajo $(\gamma=.02, \mathrm{t}=.31)$, ni la relación entre Agotamiento Emocional e Inclinación al Absentismo $(\beta=.11, \mathrm{t}=1.74)$. En base a estos resultados se reespecificó de nuevo el modelo para ambas muestras eliminando las relaciones no significativas. En la Figura 2 se representa el modelo reelaborado para la muestra de hombres y en la Figura 3 el modelo reelaborado para la muestra de mujeres.

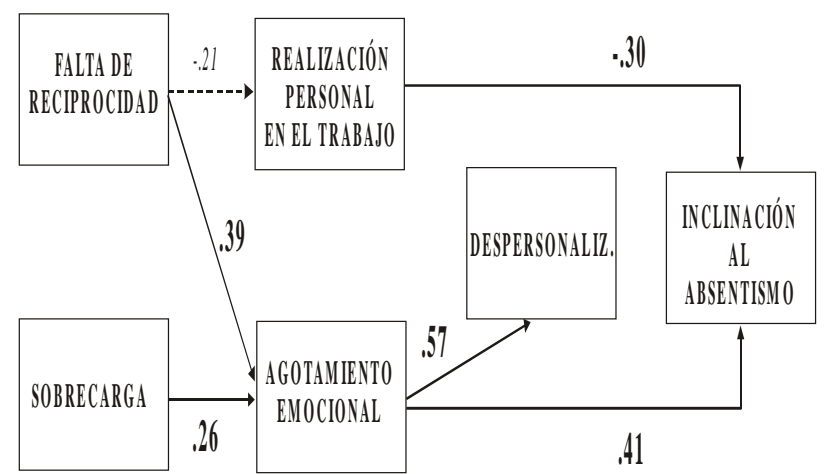

Figura 2. Modelo con los coeficientes estructurales estimados después de eliminar las relaciones no significativas en la muestra de hombres.

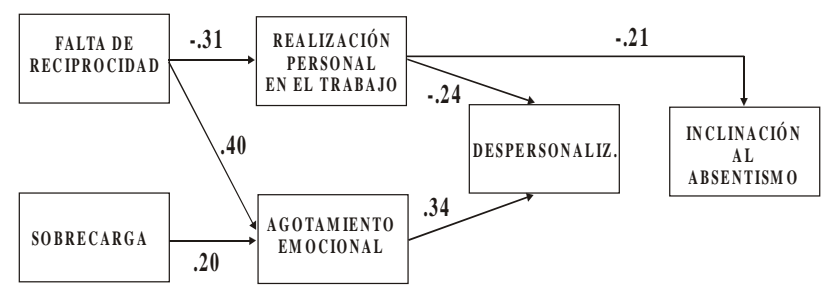

Figura 3. Modelo con los coeficientes estructurales estimados después de eliminar las relaciones no significativas en la muestra de mujeres.
Como se puede observar en la Tabla 3, el modelo para la muestra de hombres ajustó adecuadamente a los datos según todos los índices de ajuste global considerados, mientras que en la muestra de mujeres el ajuste adecuado del modelo quedó establecido por los índices GFI y CFI. Todas las relaciones restantes entre las variables resultaron significativas, salvo la relación Falta de Reciprocidad-Realización Personal en el Trabajo que pasó a ser no significativa en la muestra de hombres $(\gamma=-.21, \mathrm{t}=-1.82)$. Los efectos directos significativos quedaron de la siguiente manera: Falta de Reciprocidad presentó un efecto directo significativo sobre Agotamiento Emocional similar en ambas muestras (hombres: $\gamma=.39$, mujeres $\gamma=.40$ ), pero su efecto sobre Realización Personal en el Trabajo resultó significativo sólo en la muestra de mujeres, aunque el valor del parámetro para la muestra de hombres $(\gamma=-.21, \mathrm{t}<1.96)$ fue similar a otros parámetros significativos de la muestra de mujeres. Sobrecarga laboral presentó efectos directos significativos sobre Agotamiento Emocional en ambas muestras (hombres: $\gamma=.26$, mujeres $\gamma=.20$ ). Realización Personal en el Trabajo tuvo efectos significativos sobre Despersonalización sólo en la muestra de mujeres $(\beta=-.24)$, y sobre Inclinación al Absentismo en ambas muestras (hombres: $\beta=-.30$, mujeres $\beta=-.21$ ). Y Agotamiento Emocional presentó efectos directos significativos sobre Despersonalización en ambas muestras (hombres: $\beta=$ .57 , mujeres $\beta=.34$ ) y sobre Inclinación al Absentismo sólo en la muestra de hombres $(\beta=.41)$.

El valor de $\mathrm{R}^{2}$ para las variables del modelo resultó diferente en función de la muestra. En la muestra de hombres, al no considerar los efectos de Falta de Reciprocidad, el valor de $\mathrm{R}^{2}$ para Realización Personal en el Trabajo resultó nulo, mientras que para las mujeres alcanzó un valor de $\mathrm{R}^{2}=.10$. Para Agotamiento Emocional, el valor resultó similar en ambas muestras (hombres: $\mathrm{R}^{2}=.29$, mujeres: $\mathrm{R}^{2}=$ .24). En Despersonalización el valor resultó superior para la muestra de hombres (hombres: $\mathrm{R}^{2}=.32$, mujeres: $\mathrm{R}^{2}=.20$ ), y en Inclinación al Absentismo también fue muy superior en la muestra de hombres (hombres: $\mathrm{R}^{2}=.28$, mujeres: $\mathrm{R}^{2}=.04$ ).

\section{DISCUSIÓN}

Como se ha planteado en la introducción el objetivo del estudio era doble. Los resultados obtenidos presentan algunos datos diferenciales. En primer lugar, el análisis de las medias de las variables permite afirmar que los profesionales de enfermería 
varones experimentan significativamente mayores niveles de falta de reciprocidad que sus colegas femeninas, y mayor inclinación al absentismo. Asimismo, sus niveles de despersonalización fueron significativamente mayores. Como ha quedado señalado en la introducción, la consideración tradicional de la profesión de enfermería como una profesión eminentemente femenina, y las diferencias en los modelos de socialización de nuestra sociedad occidental para los diferentes géneros puede ser la base para la explicación de estas diferencias. Mientras que las mujeres son condicionadas socialmente para cuidar, educar y encargarse de los niños, las tareas del hogar, atender al cónyuge o pareja, y en algunas ocasiones a los ancianos, en los hombres se enfatiza la dureza e indiferencia emocional, así como una actitud de orientación al logro opuesta a la interdependencia y habilidad en las relaciones interpersonales.

En relación al segundo objetivo planteado, el modelo presenta un ajuste adecuado a los datos según diferentes índices de ajuste global, tanto para la muestra de hombres como para la de mujeres, por lo que puede ser considerado como una forma adecuada de representar el desarrollo del proceso del síndrome de quemarse por el trabajo. En este proceso la baja realización personal en el trabajo y los niveles altos de agotamiento emocional aparecen como variables mediadoras entre las fuentes de estrés (falta de reciprocidad en los intercambios y sobrecarga laboral), la despersonalización y las actitudes hacia el absentismo de los profesionales de enfermería. Al observar las relaciones significativas entre las variables del modelo se observan similitudes y diferencias en función del género.

La sobrecarga laboral aparece como un predictor significativo de agotamiento emocional, pero no de realización personal en el trabajo, en el caso de ambos sexos. Este resultado apoya los resultados obtenidos en estudios de metaanálisis (Lee y Ashforth, 1996), y en estudios con profesionales de enfermería (Janssen et al., 2000; Richardsen et al., 1992; Zellars, et al., 2000), y confirma que la percepción de sobrecarga laboral genera preferentemente una respuesta de carácter emocional. La falta de reciprocidad percibida aparece como predictor de agotamiento emocional para ambos sexos, pero sólo resultó predictor significativo de realización personal en el trabajo en la muestra de mujeres. No obstante, y dado el tamaño del parámetro en la muestra de hombres (gamma $=-.21$ ), este resultado puede estar condicionado por el tamaño de la muestra más que por el género de los sujetos (ver Byrne, 1998, p. 104).
Respecto a la relación entre las dimensiones del MBI, para la muestra de mujeres los resultados vendrían a apoyar el modelo propuesto por Gil-Monte y colaboradores (Gil-Monte y Peiró, 1997; Gil-Monte et al., 1998) según el cual la despersonalización es una estrategia de afrontamiento desarrollada frente a los sentimientos crónicos de baja realización personal en el trabajo y de agotamiento emocional. La relación entre agotamiento emocional y despersonalización significativa para ambos sexos confirma la solidez de esta relación entre las dimensiones del MBI. Sin embargo, el hecho de que la relación entre realización personal en el trabajo y despersonalización no sea significativa para la muestra de hombres y sí para la de mujeres sugiere que esta relación puede estar modulada por el sexo, de manera que los estudios en los que la muestra está formada por un gran porcentaje de mujeres y mínimo de hombres obtienen que la relación resulta significativa (Manassero, et al., 2000; Manzano y Ramos, 2000), pero en el caso inverso la relación tiende a ser no significativa (Durán, 2001). Esta conclusión es una hipótesis a estudiar en futuros estudios.

Por último, a la hora de estudiar el síndrome de quemarse por el trabajo en profesionales de enfermería y sus consecuencias sobre la organización, parece adecuado considerar dos padrones diferenciados, uno para los profesionales de sexo masculino y otro para los de sexo femenino. Mientras que en el grupo de mujeres la varianza en la inclinación al absentismo apenas viene explicada por los estresores considerados y por las dimensiones del síndrome $\left(\mathrm{R}^{2}=.04\right)$, en el grupo de hombres los porcentajes de varianza son manifiestamente mayores, y dignos de tomarse en cuenta $\left(\mathrm{R}^{2}=.28\right)$. Estos resultados deben ser considerados para futuros estudios en los que intervengan muestras de profesionales de enfermería, pues estas muestras están compuestas en gran medida por mujeres lo cual puede enmascarar la realidad para los profesionales de sexo masculino.

Por lo que respecta a las implicaciones prácticas del estudio, los gestores de recursos humanos en el sector ocupacional de enfermería han de tener en consideración estos resultados. Es necesario que para diseñar medidas de intervención y prevención del síndrome y del absentismo en enfermería consideren la existencia de ambos sexos en el seno de la profesión, pues desarrollan padrones de respuesta al estrés laboral diferentes. Además, la sobrecarga laboral y la percepción de equidad son variables que deben ser tenidas en cuenta para prevenir el desarrollo del síndrome de quemarse por el 
trabajo y para disminuir la tasa de absentismo en los profesionales de enfermería. Junto a ello, los resultados recomiendan evaluar y prevenir el síndrome de quemarse por el trabajo pues, dada su función mediadora entre las fuentes de estrés y sus consecuencias, su prevención disminuirá la tasa de absentismo en este sector ocupacional.

\section{REFERÊNCIAS BIBLIOGRÁFICAS}

Bacharach, S. B., Bamberger, P., y Conley, S. (1991). Workhome conflicts among nurses and engineers: mediating the impact of role stress on burnout and satisfaction at work. Journal of Organizational Behavior. 12 (3), 39-53.

Bakker, A. B., Schaufeli, W. B., Sixma, H. J., Bosveld, W., y Van Dierendonck, D. (2000). Patient demands, lack of reciprocity, and burnout: A five-year longitudinal study among general practitioners. Journal of Organizational Behavior. 21, 425-441.

Bentler, P. M. (1992). On the fit of models to covariances and methodology to the Bulletin. Psychological Bulletin. 112, 400-404.

Browne, M. W., y Cudeck, R. (1993). Alternative ways of assessing model fit. En K. A. Bollen y J. S. Long (Eds.), Testing structural equation models (pp. 136-162). Newbury Park, CA: Sage.

Buunk, B. P., y Schaufeli, W. B. (1993). Burnout: A perspective from social comparison theory. En W. B. Schaufeli, C. Maslach y T. Marek (Eds.), Professional Burnout: Recent Developments in Theory and Research (pp. 53-69). London: Taylor \& Francis.

Byrne, B. M. (1992). The Maslach Burnout Inventory: Validating factorial structure and invariance across intermediate, secondary, and university educators. Multivariate Behavioral Research. 26 (4), 583-605.

Byrne, B. M. (1998). Structural equation modeling with LISREL, PRELIS, and SIMPLIS: Basic concepts, applications, and programming. London: Lawrence Erlbaum Associates.

Caplan, R. D., Cobb, S., French, J. R., Van Harrison, R., y Pinneau, S. R. (1975). Job demands and worker health. Ann Arbor, Michigan: Institute for Social Research.

Donnerstein, E. y Hatfield, E. (1982). Aggression and inequity. En J. Greenberg y R. L. Cohen (Eds.), Equity and justice in social behavior (pp. 309-336). New York: Academic Press.

Durán, M. A. (2001). El síndrome de burnout en organizaciones policiales: una aproximación secuencial. Tesis doctoral no publicada, Facultad de Psicología, Universidad de Málaga, España.

Firth, H., y Britton, P. (1989). Burnout, absence and turnover amongst British nursing staff. Journal of Occupational Psychology. 62 (1), 55-59.

FOREM-CCOO (2000). Estudio sobre las necesidades de formación en prevención de riesgos psicosociales para delegados y delegadas de prevención. Madrid: FOREM-CCOO.
Freudenberger, H. J. (1974). Staff burn-out. Journal of Social Issues. 30 (1), 159-165.

Gil-Monte, P. R., y Peiró, J. M. (1997). Desgaste psíquico en el trabajo: el síndrome de quemarse. Madrid: Síntesis.

Gil-Monte, P. R., Peiró, J. M. y Valcárcel, P. (1996). Influencia de las variables de carácter sociodemográfico sobre el síndrome de burnout: un estudio en una muestra de profesionales de enfermería. Revista de Psicología Social Aplicada. 6 (2), 43-63.

Gil-Monte, P. R., Peiró, J. M., y Valcárcel, P. (1998): A model of burnout process development: An alternative from appraisal models of stress. Comportamento Organizacional e Gestão. 2 (2), 211-220.

Golembiewski, R. T., Munzenrider, R., y Carter, D. (1983). Phases of progressive burnout and their work site covariants: Critical issues in OD research and praxis. Journal of Applied Behavioral Science. 19 (4), 461-481.

Hair, J. H., Anderson, R. E., Tatham, R. L., y Black, W. C. (1995). Multivariate data analysis (4th ed.). Englewood Cliffs, NJ: Prentice-Hall.

Hoyle, R. H. (1995). The Structural Equation Modeling approach: Basic concepts and fundamental issues. En R. H. Hoyle (Ed.), Structural Equation Modeling: Concepts, issues, and applications (pp. 1-15). Thousand Oaks, CA: Sage.

Iverson, R. D., Olekalns, M., y Erwin, P. J. (1998). Affectivity, organizational stressors, and absenteeism: A causal model of burnout and its consequences. Journal of Vocational Behavior. 52, 1-23.

Janssen, P. P., Schaufeli, W. B., y Houkes, I. (1999). Workrelated and individual determinants of the three burnout dimensions. Work \& Stress. 13(1), 74-86.

Jöreskog, K. G., y Sörbom, D. (1996). LISREL 8: User's reference guide. Chicago: Scientific Software International.

Kelley, H. H. (1979). Personal Relationships: Their Structures and Processes. Hillsdale, N.J.: Erlbaum.

Lee, R. T., y Ashforth, B. E. (1993a). A further examination of managerial burnout: Toward an integrated model. Journal of Organizational Behavior. 14, 3-20.

Lee, R. T., y Ashforth, B. E. (1993b). A longitudinal study of burnout among supervisors and managers: Comparisons between the Leiter and Maslach (1988) and Golembiewski et al. (1986) models. Organizational Behavior and Human Decision Processes. 54, 369-398.

Lee, R. T., y Ashforth, B. E. (1996). A meta-analytic examination of the correlates of the three dimensions of job burnout. Journal of Applied Psychology. 81 (2), 123-133.

Leiter, M. P. (1991). Coping patterns as predictors of burnout: The function of control and escapist coping patterns. Journal of Organizational Behaviour. 12 (2), 123-144.

Leiter, M. P., y Maslach, C. (1988). The impact of interpersonal environment on burnout and organizational commitment. Journal of Organizational Behavior. 9, 297-308.

Manassero, M. A., García, E., Vázquez, A., Ferrer, V. A., Ramis, C., y Gili, M. (2000). Análisis causal del burnout en la enseñanza. Revista de Psicología del Trabajo y de las Organizaciones. 16 (2), 173-195. 
Manzano, G., y Ramos, F. (2000). Enfermería hospitalaria y síndrome de burnout. Revista de Psicología del Trabajo y de las Organizaciones. 16 (2), 197-213.

Marsh, H. W., Balla, J. R., y Hau, K. T. (1996). An evaluation of Incremental Fit Indices: A clarification of mathematical and empirical properties. En G. A. Marcoulides y R. E. Schumacker (Eds.), Advanced structural equation modeling: issues and techniques (pp. 315-353) Mahwah, NJ: Erlbaum.

Maslach, C., y Jackson, S. E. (1986). Maslach Burnout Inventory (20 ed, 198110 ed.). Palo Alto, California: Consulting Psychologists Press.

McClintock, C. G. y Keil, L. J. (1982). Equity and social exchange. En J. Greenberg y R. L. Cohen (Eds.), Equity and justice in social behavior (pp. 337-387). New York: Academic Press.

Moreno, B. (2000). Olvido y recuperación de los factores psicosociales en la salud laboral. Archivo de Prevención de Riesgos Laborales. 3 (1), 3-4.

Novak, M., y Chappell, N. L. (1996). The impact of cognitively impaired patients and shift on nursing assistant stress. International Journal of Aging and Human Development. 43 (3), 235-248.

Richardsen, A. M., Burke, R. J., y Leiter, M. P. (1992). Occupational demands, psychological burnout and anxiety among hospital personnel in Norway. Anxiety, Stress and Coping. 5 (1), 55-68.

SATSE (1999). Guía para la prevención del estrés. Madrid: SATSE.

Schaufeli, W. B., y Janczur, B. (1994). Burnout among nurses: A Polish-Dutch comparison. Journal of Cross-Cultural Psychology. 25 (1), 95-113.

Van Dierendonck, D., Schaufeli, W. B., y Buunk, B. P. (1998). The evaluation of an individual intervention program: The role of inequity and social support. Journal of Applied Psychology. 83 (3), 392-407.

Zellars, K. L., Perrewé, P. L., y Hochwarter, W. A. (2000). Burnout in health care: The role of the Five Factor of personality. Journal of Applied Social Psychology. 30 (8), 1570-1598.

Zohar, D. (1997). Predicting burnout with a hassle-based measure of role demands. Journal of Organizational Behavior. 18, 101-115.

Recebido em 01/04/2002

Revisado em 07/05/2002

Aceito em 10/05/2002 